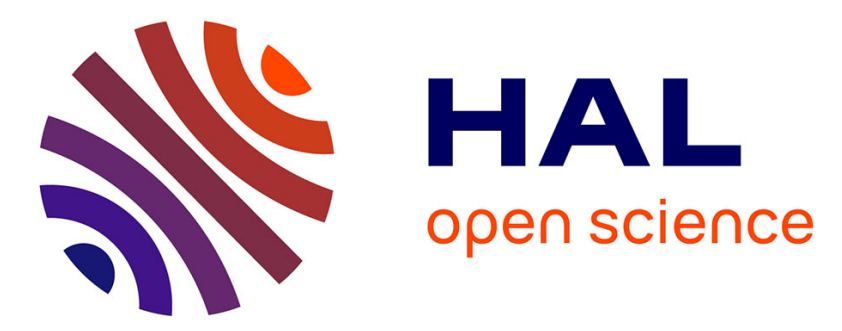

\title{
A Conservative Coupling Method for Fluid-Structure Interaction in the Compressible Case
}

Laurent Monasse, Virginie Daru, Christian Mariotti, Serge Piperno, Christian

Tenaud

\section{- To cite this version:}

Laurent Monasse, Virginie Daru, Christian Mariotti, Serge Piperno, Christian Tenaud. A Conservative Coupling Method for Fluid-Structure Interaction in the Compressible Case. Sixth International Conference on Computational Fluid Dynamics, ICCFD6, Jul 2010, St Petersburg, Russia. pp.473-478, 10.1007/978-3-642-17884-9_59. hal-00676721

\section{HAL Id: hal-00676721 \\ https://hal-enpc.archives-ouvertes.fr/hal-00676721}

Submitted on 19 Dec 2018

HAL is a multi-disciplinary open access archive for the deposit and dissemination of scientific research documents, whether they are published or not. The documents may come from teaching and research institutions in France or abroad, or from public or private research centers.
L'archive ouverte pluridisciplinaire HAL, est destinée au dépôt et à la diffusion de documents scientifiques de niveau recherche, publiés ou non, émanant des établissements d'enseignement et de recherche français ou étrangers, des laboratoires publics ou privés. 


\title{
A Conservative Coupling Method for Fluid- Structure Interaction in the Compressible Case
}

\author{
Laurent Monasse, Virginie Daru, Christian Mariotti, and Serge Piperno
}

\begin{abstract}
We propose a general explicit coupling method between a Finite Volume method for compressible $\mathrm{fl} \mathrm{w}$ and a rigid body. The coupling strategy is based on the idea of Embedded Boundary methods (Pember et al., J. Comput. Phys. 120:278$304,1995)$. The flu es are computed everywhere in the Cartesian grid, and are modifie at the solid boundaries to enforce flui mass conservation. The coupling between the flui and the solid is designed to ensure a balance in momentum and energy. We prove the exact numerical conservation of several simple uniform $\mathrm{fl}$ ws. An illustrative example of the liftoff of a cylinder by a shock wave is presented and compared with existing results.
\end{abstract}

\section{Introduction}

For fluid-structur interaction problems, two main types of methods were developed. The Arbitrary Lagrangian-Eulerian method (ALE) was firs developed in the late seventies [4]. However, as a body-fitte method, the ALE technique requires remeshing of the flui domain when the solid is subjected to large deformations or breaking, which can be computationally demanding. In order to avoid bodyfittin and remeshing, Peskin [14] proposed the Immersed Boundary method for the coupling of incompressible biological flui fl ws with moving elastic boundaries. Penalization type method have been applied to compressible fluid-structur interaction [1], but the stiff condition on the time-step as the penalization parameter is increased is unsatisfactory. Direct forcing methods, which modify the values of the flui cells in the vicinity of the solid boundary, have been widely applied $[6,10,12]$, but still do not ensure the conservation of physical quantities at the interface.

\footnotetext{
L. Monasse $(\bowtie)$

Université Paris-Est, CERMICS, 77455 Marne-la-Vallée, France

e-mail: monassel@cermics.enpc.fr
} 
Conservative methods for Finite Volumes with complex geometries have been developed, such as the Embedded Boundary method [13]. Several procedures were proposed to avoid the stability condition restriction due to cut cells with small volume. We adopt here the solution consisting in merging the small cut-cells with their stable neighbors, that were successfully applied for compressible fluid-structur interaction [8].

In the sequel the equations for flu modificatio remain similar to [8]. The main difference lies in the coupling approach. Ultimately, our aim is to couple the compressible fl w with a Discrete Element method for the solid. As the Discrete Element method is computationally expensive, we use an explicit coupling method. In this paper, we restrict ourselves to rigid solids, but the method also applies for deformable bodies. The algorithm is carried out in a partitioned way: the flui pressure makes the solid move, and the updated position of the solid induces a modificatio of the flui flu es at the new boundary. The flui solver is based on the time splitting high order scheme developed in [3]. However the theoretical analysis of the coupling algorithm does not depend on the scheme that is used.

\section{Description of the Method}

The position of the solid in the flui domain is taken into account using the Embedded Boundary method $[5,7-9,13]$. At time $t$, the solid occupies a volume fraction $\alpha_{i}$ of cell $i$, and all variables are assumed to be uniform in the cell. The conservative quantities contained in the cell are therefore equal to their value at the center of the cell times the volume of the cell and the volume fraction of flui $1-\alpha_{i}$. In the same way, the computed flu es are assumed to be constant on cell faces. If we denote $\kappa_{i j}$ the surface fraction of the face between cells $i$ and $j$ occupied by solid, we set the effective flu between $i$ and $j$ as the computed flu times the surface of their interface and the surface fraction of flui $1-\kappa_{i j}$. Additional flu es come from the presence of the solid boundary, and are computed in order to yield exact conservation of flui mass and of total momentum and energy of the system.

We now describe the algorithm we developed, which preserves the fully conservative properties of the Finite Volume method combined with the Embedded Boundary method. At the beginning of a time step, at time $n \Delta t$, the known quantities are:

- The position of the center of mass of the solid particle $\mathbf{X}^{n}$ and the rotation matrix $\mathrm{Q}^{n}$

- The velocity of the center of mass of the solid particle $\mathbf{V}^{n}$ and the angular quantity of movement matrix $\mathrm{P}^{n}$

- The flui state: density $\varrho^{n}$, velocity $\mathbf{u}^{n}$ and pressure $p^{n}$.

The general algorithm is as follows: 


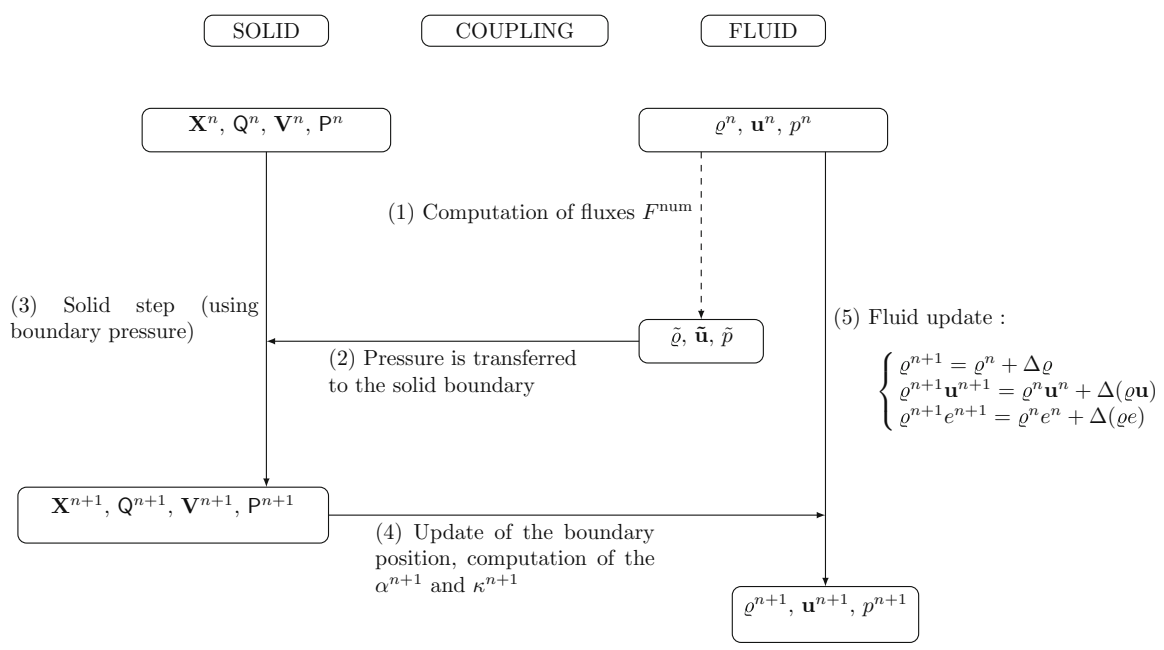

The pressures $p_{x}^{\text {ref }}$ and $p_{y}^{\text {ref }}$ transferred to the solid boundary are chosen to be the temporal average of the reference pressures used during the partial steps of the flu computation (1) in the $x$ and $y$ directions (in our case, due to the splitting method; these steps could also be the steps of a Runge-Kutta method). The solid is advanced in step (3) using a classical second order Verlet scheme for translation and a second order RATTLE scheme for rotation. Details can be found in [11]. The solid rigid body is supposed to be of general polygonal shape, and the computation of intersections in step (4) is straightforward.

Let the indices $l, r, b$ and $t$ denote the respective left, right, bottom and top faces of a cell $\mathscr{C}$. Following [8], the cut-cells computation in step (5) is written as follows in the 2D case:

- For each solid boundary $\mathscr{F}$, compute the quantity $\Delta w_{\mathscr{F}}^{n}$ swept by boundary $\mathscr{F}$, such that :

$$
\sum_{\mathscr{F}} \Delta w_{\mathscr{F}}^{n}=\sum_{\mathscr{C}}\left(\alpha_{\mathscr{C}}^{n+1}-\alpha_{\mathscr{C}}^{n}\right) w_{\mathscr{C}}^{n}
$$

where $w$ denotes alternately density $\varrho$, momentum $\varrho \mathbf{u}$ or energy $\varrho e$. In practice, we compute $\Delta w_{\mathscr{F}}^{n}$ as the integral of $w^{n}$ in the quadrangle define by the positions of $\mathscr{F}$ at time $n \Delta t$ and $(n+1) \Delta t$.

- Compute the numerical flu es $F_{\mathscr{F}}^{\text {num }}$ at the solid boundary using $p_{x}^{\text {ref }}$ and $p_{y}^{\text {ref }}$ stored in the boundary $\mathscr{F}$. The flu es are given by:

- If $w=\varrho, F_{\mathscr{F}}^{\text {num }}=0$

- If $w=\varrho u, F_{\mathscr{F}}^{\mathrm{num}}=\frac{1}{S_{\mathscr{F}}} \int_{\mathscr{F}} p_{x}^{\mathrm{ref}} n_{\mathscr{F}}^{x}$ 


$$
\begin{aligned}
& \text { - If } w=\varrho v, F_{\mathscr{F}}^{\mathrm{num}}=\frac{1}{S_{\mathscr{F}}} \int_{\mathscr{F}} p_{y}^{\mathrm{ref}} n_{\mathscr{F}}^{y} \\
& - \text { If } w=\varrho e, F_{\mathscr{F}}^{\mathrm{num}}=\frac{1}{S_{\mathscr{F}}} \mathbf{V}_{\mathscr{F}}^{n+\frac{1}{2}} \cdot \int_{\mathscr{F}}\left(\begin{array}{c}
p_{x}^{\mathrm{ref}} n_{\mathscr{F}}^{x} \\
p_{y}^{\mathrm{ref}} n_{\mathscr{F}}^{y}
\end{array}\right)
\end{aligned}
$$

- Compute $\Delta w_{\mathscr{C}}$ for each cell $\mathscr{C}$ :

$$
\begin{aligned}
\left(1-\alpha_{\mathscr{C}}^{n+1}\right) \Delta w_{\mathscr{C}}=\Delta t & \left(\frac{1-\kappa_{\mathscr{C} l}^{n+1}}{\Delta x} F_{\mathscr{C} l}^{\mathrm{num}}-\frac{1-\kappa_{\mathscr{C} r}^{n+1}}{\Delta x} F_{\mathscr{C} r}^{\mathrm{num}}+\frac{1-\kappa_{\mathscr{C} b}^{n+1}}{\Delta y} F_{\mathscr{C} b}^{\mathrm{num}}\right. \\
& \left.-\frac{1-\kappa_{\mathscr{C} t}^{n+1}}{\Delta y} F_{\mathscr{C} t}^{\mathrm{num}}\right)+\sum_{\mathscr{F} \in \mathscr{C}} \frac{\Delta t S_{\mathscr{F}}}{\Delta x \Delta y} F_{\mathscr{F}}^{\mathrm{num}}+\sum_{\mathscr{F} \in \mathscr{C}} \Delta w_{\mathscr{F}}^{n}
\end{aligned}
$$

- Update the value of $w_{\mathscr{C}}$ in every cell :

$$
w_{\mathscr{C}}^{n+1}=w_{\mathscr{C}}^{n}+\Delta w_{\mathscr{C}}
$$

To ensure stability of the method for small cut-cells, we follow the procedure described in [8], with minor changes due to the impossibility to defin a normal vector in a cell occupied by two boundaries. We defin small cells as $\alpha_{\mathscr{C}}>0.5$. If we mix cell $\mathscr{C}$ with a target cell $\mathscr{C}_{t}$, so that the fina value $w$ of the two cells is equal, we have to exchange the quantities $M_{\mathscr{C} \mathscr{C}_{t}}=\frac{\alpha_{\mathscr{C}}}{\alpha_{\mathscr{C}}+\alpha_{\mathscr{C}}}\left(w_{\mathscr{C}_{t}}-w_{\mathscr{C}}\right)$ and $M_{\mathscr{C}_{t} \mathscr{C}}=$ $\frac{\alpha_{\mathscr{C}}}{\alpha_{\mathscr{C}}+\alpha_{\mathscr{C}} t}\left(w_{\mathscr{C}}-w_{\mathscr{C}_{t}}\right)$, and we easily check that $w_{\mathscr{C}}+M_{\mathscr{C}_{\mathscr{C}}}=w_{\mathscr{C}_{t}}+M_{\mathscr{C}_{t} \mathscr{C}}$. In the 2D case, we have to make a choice for the target cell $\mathscr{C}_{t}$. We f x $\mathscr{C}_{t}$ to be the fully-flui cell $\left(\alpha_{\mathscr{C}_{t}}=0\right)$ nearest to cell $\mathscr{C}$, such that the path between the two cells does not cross a solid boundary. A recursive subroutine find such a target cell after few iterations.

Let us note that the mixing procedure is entirely conservative, and ensures that the significan volume for a cell is consistent with the usual CFL condition based on standard cell size.

\section{Theoretical Results}

The following results were theoretically proven:

\section{- Mass, momentum and energy conservation:}

When there is no infl $\mathrm{w}$ into or outfl $\mathrm{w}$ from the domain, conservation of flui mass is ensured. For periodic boundary conditions, the momentum is exactly balanced between flui and solid during each time-step. For periodic or reflectin boundary conditions, the energy received by the flui from the solid is exactly balanced by the work of flui pressure forces on the solid during each time-step. 


\section{- Frame indifference:}

Let an arbitrary shaped rigid body moving at constant velocity and no rotation, be immersed in a uniform flui $\mathrm{fl}$ wing at the same velocity. The uniform motion of both the solid and the flui is preserved by the coupling algorithm.

\section{- Free slip along a straight boundary:}

A uniform $\mathrm{fl} \mathrm{W}$ parallel to a rigid semi-infinit half-plane is preserved by the coupling algorithm.

The last result shows that no numerical boundary layer or artificia boundary roughness appear at the solid boundary, even when it is not aligned with the Cartesian mesh.

\section{Numerical Results}

Due to space limitations, we only present here a moving boundary benchmark that was firs proposed in [7] and also treated in [8]. A rigid cylinder of density $7.6 \mathrm{~kg}$ $\mathrm{m}^{-3}$, initially resting on the lower wall of a two-dimensional channel fille with air at standard conditions, is driven and lifted upwards by a Mach 3 shock wave. The results obtained on a 1,600 × 320 grid are shown in Fig. 1. We observe good agreement with the results shown in $[2,8]$. Small differences in the position of the shock waves can be noticed but no reference solution exists for this case. We also observe a strong vortex under the cylinder which is much weaker in [8]. This vortex seems to be associated with a Kelvin-Helmholtz instability originating at the contact discontinuity present below the cylinder.

In addition, when considering the fina position of the center of mass of the cylinder, we observe a fast grid convergence of the method. The position we obtain on a $400 \times 80$-grid is comparable to that obtained on a $1,600 \times 320$-grid in [8]. With increasing resolution on grids $400 \times 80,800 \times 160$ and $1,600 \times 320$ [8] gives
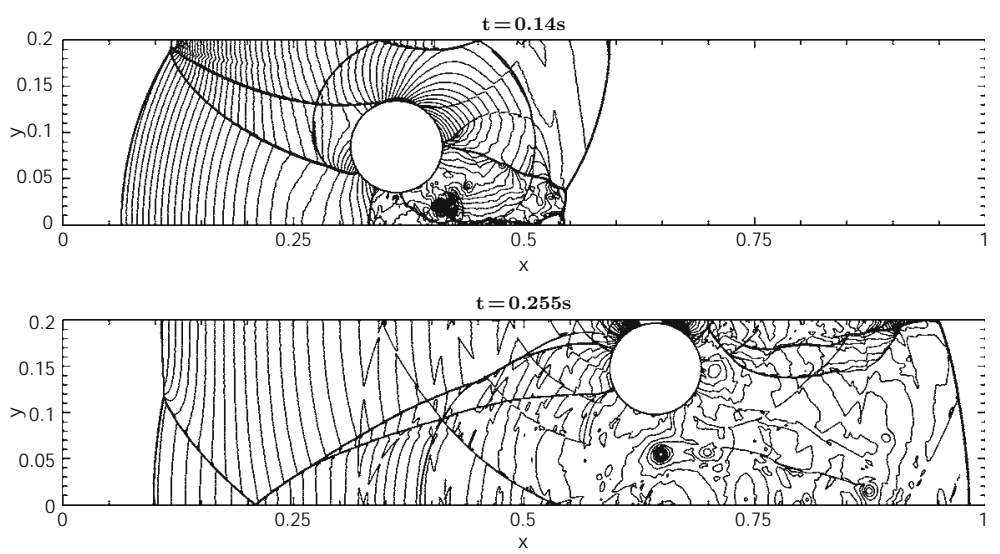

Fig. 160 contours of flui pressure from 0 to 28 at different times, $\Delta x=\Delta y=6.25 \times 10^{-4}$ 
positions $(0.659,0.132),(0.649,0.145)$ and $(0.641,0.147)$; on the same grids, we obtain $(0.64375,0.1463),(0.64278,0.1471)$ and $(0.64253,0.1471)$.

\section{References}

1. Aquelet, N., Souli, M., Olovsson, L.: Euler-lagrange coupling with damping effects: Application to slamming problems. Comput. Methods Appl. Mech. Eng. 195(1-3), 110-132 (2006)

2. Arienti, R., Hung, P., Morano, E., Shepherd, J.E.: A level set approach to Eulerian-Lagrangian coupling. J. Comput. Phys. 185, 213-251 (2003)

3. Daru, V., Tenaud, C.: High order one-step monotonicity-preserving schemes for unsteady compressible fl w calculations. J. Comput. Phys. 193(2), 563-594 (2004)

4. Donea, J., Giuliani, S., Halleux, J.P.: An arbitrary Lagragian Eulerian finit element method for transient dynamic fluid-structur interactions. Comput. Methods Appl. Mech. Eng. 33, 689-723 (1982)

5. Dragojlovic, Z., Najmabadi, F., Day, M.: An embedded boundary method for viscous, conducting compressible fl w. J. Comput. Phys. 216(1), 37-51 (2006)

6. Fadlun, E.A., Verzicco, R., Orlandi, P., Mohd-Yusof, J.: Combined immmersed-boundary finite-di ference methods for three-dimensional complex fl w simulations. J. Comput. Phys. 161(1), 35-60 (2000)

7. Falcovitz, J., Alfandary, G., Hanoch, G.: A two-dimensional conservation laws scheme for compressible fl ws with moving boundaries. J. Comput. Phys. 138, 83-102 (1997)

8. Hu, X.Y., Khoo, B.C., Adams, N.A., Huang, F.L.: A conservative interface method for compressible fl ws. J. Comput. Phys. 219(2), 553-578 (2006)

9. Miller, G.H., Colella, P.: A conservative three-dimensional Eulerian method for coupled solidflui shock capturing. J. Comput. Phys. 183(1), 26-82 (2002)

10. Mohd-Yusof, J.: Combined immersed-boundary/b-spline methods for simulation of $\mathrm{fl} \mathrm{w}$ in complex geometries. CTR Annual Research Briefs, Center for Turbulence Research, NASA Ames/Stanford University (1997)

11. Monasse, L., Mariotti, C.: An energy-preserving discrete element method for elastodynamics. (submitted to ESAIM journal "Mathematical Modelling and Numerical Analysis")

12. De Palma, P.M., de Tullio, D., Pascazio, G., Napolitano, M.: An immersedboundary method for compressible viscous fl ws. Comp. Fluid 35(7), 693-702 (2006)

13. Pember, R.B., Bell, J.B., Colella, P., Crutchfield W.Y., Welcome, M.L.: An adaptive Cartesian grid method for unsteady compressible fl $\mathrm{w}$ in irregular regions. J. Comput. Phys. 120, 278-304 (1995)

14. Peskin, C.S.: Numerical analysis of blood $\mathrm{fl} \mathrm{w}$ in the heart. J. Comput. Phys. 25, 220-252 (1977) 